\title{
Spin relaxation and combined resonance in two-dimensional electron systems with spin-orbit disorder
}

\author{
V. K. Dugaev ${ }^{1,2}$, E. Ya. Sherman ${ }^{3,4}$, V. I. Ivanov ${ }^{5}$, and J. Barnaś 6,7 \\ ${ }^{1}$ Department of Physics, Rzeszów University of Technology, \\ Powstańców Warszawy 6, 35-959 Rzeszów, Poland \\ ${ }^{2}$ Department of Physics and CFIF, Instituto Superior Técnico, \\ TU Lisbon, Av. Rovisco Pais 1049-001 Lisbon, Portugal \\ 3 Basque Foundation for Science IKERBASQUE, \\ Alameda Urquijo 36-5, 48011, Bilbao, Bizkaia, Spain \\ ${ }^{4}$ Department of Physical Chemistry, Universidad del País Vasco, Bilbao, 48080 Bizkaia, Spain \\ ${ }^{5}$ Insitute for Problems of Materials Science, Ukrainian Academy of Sciences, Vilde 5, 58001 Chernovtsy, Ukraine \\ ${ }^{6}$ Institute of Molecular Physics, Polish Academy of Sciences, Smoluchowskiego 17, 60-179 Poznań, Poland \\ 7 Department of Physics, Adam Mickiewicz University, Umultowska 85, 61-614 Poznań, Poland
}

(Dated: November 6, 2018)

\begin{abstract}
Disorder in spin-orbit (SO) coupling is an important feature of real low-dimensional electron structures. We study spin relaxation due to such a disorder as well as resulting abilities of spin manipulation. The spin relaxation reveals quantum effects when the spatial scale of the randomness is smaller than the electron wavelength. Due to the disorder in SO coupling, a time-dependent external electric field generates a spatially random spin-dependent perturbation. The resulting electric dipole spin resonance in a two-dimensional electron gas leads to spin injection in a frequency range of the order of the Fermi energy. These effects can be important for possible applications in spintronics.

PACS numbers: $72.25 . \mathrm{Rb}, 72.25 . \mathrm{Hg}$
\end{abstract}

Electron dynamics in low-dimensional semiconductor structures reveals features of a spin dependent transport that are interesting for fundamental and applied research $^{1}$. One of the main ingredients necessary to generate spin dependent transport in nonmagnetic semiconductor systems is the SO interaction. Such an interaction offers a possibility of an efficient and fast spin manipulation with electric fields, which in turn allows to prepare a required spin state $2,3,4,5,6,7,8$ At the same time, spin relaxation and decoherence due to the $\mathrm{SO}$ coupling prevent long-distance spin propagation. Two models are widely used to describe the SO coupling in lowdimensional structures: the Rashba and the Dresselhaus ones. In both models, the SO field and the corresponding spin precession rate are approximately linear in the electron momentum. Random evolution in the momentum due to collisions with impurities, phonons, and other electrons results in randomness in the spin precession, and thus leads to spin relaxation. However, in reality both interactions have an intrinsic randomness due to system imperfections, including the fluctuations in the dopant ion density ${ }^{9.10}$ or random bonds at the quantum well $(\mathrm{QW})$ interface ${ }^{11}$. Even if the mean values of the Rashba and Dresselhaus fields vanish, their fluctuations remain and can cause interesting consequences, including memory effects $\frac{10}{}$, spin Hall effect in the finite-size systems $\frac{12}{}$, and spin-dependent localization. $\stackrel{13}{\underline{n}}$

There are at least four different two-dimensional (2D) systems, where the SO disorder plays an important or crucial role. First, the effect of random SO coupling can be responsible for the spin relaxation in $\mathrm{Si} / \mathrm{Ge}$ QWs $\underline{11,14}$ Second, the spin-dependent disorder influences $\frac{15}{\underline{5}}$ the spin helix pattern recently observed in the GaAs (001)
QW with the balanced Rashba and Dresselhaus terms $\stackrel{16}{\underline{1 n}}$ Third, the randomness causes relaxation of the spin component along the growth axis observed in Ref. 17] in GaAs (011) QW, investigated now for spintronics applications ${ }^{17,18}$ Fourth, the most recent example of the system with random SO coupling is graphene, where the randomness and spin relaxation appear due to the rippling of the layers $\frac{19}{9}$ and due to the disorder and electronphonon coupling in the substrate $\underline{\underline{20}}$

In this paper we study the effects of randomness on the spin relaxation and spin injection. We show that spin relaxation reveals interesting quantum effects arising from the non-commutativity of the momentum and coordinate-dependent randomness. The calculated spin injection can be observed in a wide range of frequencies, extended up to the electron Fermi energy.

Model. We consider a two-dimensional electron gas with fluctuating Rashba SO interaction. In the absence of external fields, the Hamiltonian has the form (we use units with $\hbar=1) H=H_{0}+H_{\text {so }}$, where

$$
\begin{aligned}
& H_{0}=-\frac{\nabla^{2}}{2 m}+U(\mathbf{r}) \\
& H_{\text {so }}=-\frac{i}{2} \sigma_{x}\left\{\nabla_{y}, \lambda(\mathbf{r})\right\}+\frac{i}{2} \sigma_{y}\left\{\nabla_{x}, \lambda(\mathbf{r})\right\}
\end{aligned}
$$

Here $m$ is the electron effective mass, $U(\mathbf{r})$ is the random potential leading to the momentum relaxation time $\tau_{p}$, and $\{$,$\} stands for the anticommutator. The random$ Rashba field $\lambda(\mathbf{r})$ has zero expectation value $\langle\lambda(\mathbf{r})\rangle=0$ and correlation function

$$
C_{\lambda \lambda}\left(\mathbf{r}-\mathbf{r}^{\prime}\right) \equiv\left\langle\lambda(\mathbf{r}) \lambda\left(\mathbf{r}^{\prime}\right)\right\rangle=\left\langle\lambda^{2}\right\rangle F\left(\mathbf{r}-\mathbf{r}^{\prime}\right) .
$$




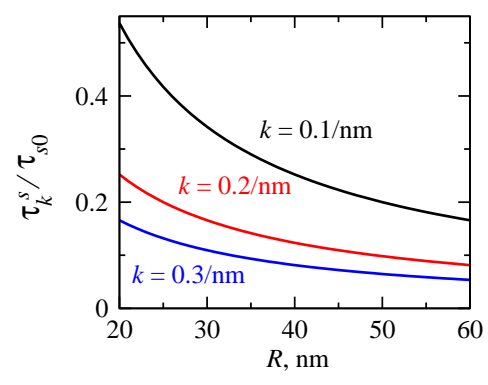

FIG. 1: (Color online) Spin relaxation time as a function of the correlation radius $R$ for different electron wave vectors $k$ marked near the lines.

The brackets $\langle\ldots\rangle$ stands for the average over the disorder, and the range function $F\left(\mathbf{r}-\mathbf{r}^{\prime}\right)$ depends on the disorder type.

Spin relaxation. We begin with calculation of spin relaxation rate due to random $H_{\text {so }}$ interaction. The eigenfunctions of Hamiltonian $H_{0}$ (normalized to the unit area) are $\psi_{\overline{\mathbf{k}}}=e^{i \mathbf{k} \cdot \mathbf{r}} \chi_{\sigma}$, where we included the spin in$\operatorname{dex} \sigma$ in the definition of momentum $\overline{\mathbf{k}}=(\mathbf{k}, \sigma)$, and $\chi_{\sigma}$ is the spin function. Matrix elements of SO interaction $V_{\overline{\mathbf{k k}^{\prime}}} \equiv\left\langle\overline{\mathbf{k}}\left|H_{\mathrm{so}}\right| \overline{\mathbf{k}}^{\prime}\right\rangle$ are

$$
V_{\overline{\mathbf{k k}^{\prime}}}=\frac{\lambda_{\mathbf{k}-\mathbf{k}^{\prime}}}{2}\left\langle\sigma\left|\sigma_{x}\left(k_{y}+k_{y}^{\prime}\right)-\sigma_{y}\left(k_{x}+k_{x}^{\prime}\right)\right| \sigma^{\prime}\right\rangle,
$$

where $\lambda_{\mathbf{k}-\mathbf{k}^{\prime}}$ is the Fourier component of the random Rashba field.

To calculate the spin relaxation time, we use the kinetic equation for spin density matrix $\rho_{\overline{\mathbf{k}}}$ (see for instance Refs. [21,22,23])

$$
\frac{\partial \rho_{\overline{\mathbf{k}}}}{\partial t}+i\left[H_{\mathrm{so}}, \rho_{\overline{\mathbf{k}}}\right]=\operatorname{St} \rho_{\overline{\mathbf{k}}} .
$$

Here, due to the absence of the regular contribution in the $H_{\text {so }}$, the commutator term in Eq.(5) vanishes. Therefore, the entire effect of $\mathrm{SO}$ randomness is included in the collision integral ${ }^{21}$

$$
\begin{aligned}
\operatorname{St} \rho_{\overline{\mathbf{k}}}=\pi \sum_{\overline{\mathbf{k}^{\prime}}}\left(2 V_{\overline{\mathbf{k k}^{\prime}}} \rho_{\overline{\mathbf{k}^{\prime}}} V_{\overline{\mathbf{k}^{\prime}} \overline{\mathbf{k}}}-V_{\overline{\mathbf{k k}^{\prime}}} V_{\overline{\mathbf{k k}^{\prime}}} \rho_{\overline{\mathbf{k}}}\right. & -\rho_{\overline{\mathbf{k}}} V_{\overline{\mathbf{k k}^{\prime}}} V_{\left.\overline{\overline{\mathbf{k}}^{\prime}} \overline{\mathbf{k}}\right)} \\
& \times \delta\left(\varepsilon_{\mathbf{k}}-\varepsilon_{\mathbf{k}^{\prime}}\right),(6)
\end{aligned}
$$

with kinetic energy $\varepsilon_{\mathbf{k}}=k^{2} / 2 m$. We take $\rho_{\overline{\mathbf{k}}}$ in the form corresponding to the only nonzero $z$-spin component $\rho_{\overline{\mathbf{k}}}=\rho_{0 \overline{\mathbf{k}}}+S_{\mathbf{k}} \sigma_{z}$, with $\rho_{0 \overline{\mathbf{k}}}$ being the equilibrium density matrix. The resulting macroscopic spin density:

$$
\left\langle s_{z}\right\rangle=\frac{1}{2} \int S_{\mathrm{k}} \frac{d^{2} k}{(2 \pi)^{2}} .
$$

Using Eqs. (4) and (6) we find

$$
\begin{array}{r}
\operatorname{St} \rho_{\overline{\mathbf{k}}}=-\frac{\pi m \sigma_{z}}{2 k} \sum_{\mathbf{q}} C_{\lambda \lambda}(\mathbf{q}) \frac{4 k^{2}-q^{2}}{q}\left(S_{\mathbf{k}^{\prime}}+S_{\mathbf{k}}\right) \\
\times \delta\left(\frac{q}{2 k}-\cos \varphi\right),
\end{array}
$$

where $C_{\lambda \lambda}(\mathbf{q})$ is the Fourier transform of the correlator $C_{\lambda \lambda}(\mathbf{r}), \varphi$ is the angle between $\mathbf{k}$ and $\mathbf{q}$, and $\mathbf{q}=\mathbf{k}-\mathbf{k}^{\prime}$ is the momentum change due to spin flip scattering by fluctuations in SO field. Since the system is macroscopically isotropic in the $x y$ plane, the coordinate-independent function $S_{\mathrm{k}}$ depends only on $k$, that yields $S_{k}=S_{k^{\prime}}$, and thus we obtain

$$
\operatorname{St} \rho_{\overline{\mathbf{k}}}=-\frac{S_{k} \sigma_{z}}{\tau_{k}^{s}},
$$

where $\tau_{k}^{s}$ is the spin relaxation time,

$$
\frac{1}{\tau_{k}^{s}}=\frac{m}{4 \pi} \int_{0}^{2 k} C_{\lambda \lambda}(\mathbf{q})\left(4 k^{2}-q^{2}\right)^{1 / 2} d q .
$$

We employ the following form of $C_{\lambda \lambda}(\mathbf{q})$ :

$$
C_{\lambda \lambda}(\mathbf{q})=2 \pi\left\langle\lambda^{2}\right\rangle R^{2} e^{-q R},
$$

where $R$ is the length scale of variations in $\lambda$. This form of correlator is realized when the Rashba SO coupling is formed by the $z$-component of electric field of random donors ${ }^{24}$ symmetrically distributed on both sides of the QW at the distance $L=R / 2$ from the QW symmetry plane.

With Eqs. (10) and (11) we obtain

$$
\begin{array}{r}
\frac{1}{\tau_{k}^{s}}=\frac{1}{2 \tau_{s 0}} \int_{0}^{2 k R} e^{-x}\left(4 k^{2} R^{2}-x^{2}\right)^{1 / 2} d x \\
=\frac{\pi R k}{2 \tau_{s 0}}\left[I_{1}(2 k R)-L_{1}(2 k R)\right],
\end{array}
$$

where $I_{1}(x)$ and $L_{1}(x)$ are the Bessel and Struve functions, respectively, and $1 / \tau_{s 0} \equiv m\left\langle\lambda^{2}\right\rangle$. As a result, we obtain

$$
\frac{1}{\tau_{k}^{s}}=\frac{1}{\tau_{s 0}} \times \begin{cases}k R, & k R \gg 1, \\ \pi(k R)^{2} / 2, & k R \ll 1 .\end{cases}
$$

Equation (13) agrees with the results of Ref.[10] for $k R \gg 1$, and shows that for given $\tau_{s 0}$ the relaxation rate rapidly decreases at small $k R$. Due to the anticommutator form of $H_{\mathrm{so}}$, at small $k R$ main contribution to the SO field comes from the derivatives of $\lambda(\mathbf{r})$. The increase in the relaxation time in this regime can be understood as a decrease in the disorder effect due to the averaging of $H_{\text {so }}$ over the area of $1 / k^{2}$. The dependence of the spin relaxation time on the correlation radius $R$ is presented in Fig. 1.

Combined resonance. Now we consider response of the system in a static magnetic field $\mathbf{B}$ to an external periodic field $\mathbf{A}(t)=\mathbf{A}_{0} e^{-i \omega t}$. The corresponding interaction $V_{\text {ext }}=-(e / c) \hat{\mathbf{v}} \mathbf{A}$, where $\mathbf{v}=i\left[H_{0}+H_{\mathrm{so}}, \mathbf{r}\right]$ is the velocity operator, induces combined resonance causing transfers between states with different spins and momenta. To study the spin dynamics, we retain only the spin-related part of the Hamiltonian and present it as:

$$
\begin{aligned}
& H=H_{0}+H_{\mathrm{so}}+H_{B}+V_{\mathrm{ext}}, \\
& H_{B}=\frac{\Delta}{2}(\boldsymbol{\sigma} \cdot \mathbf{n}), \quad V_{\mathrm{ext}}=-\lambda(\mathbf{r}) \frac{e}{c}\left(\sigma_{x} A_{y}-\sigma_{y} A_{x}\right),
\end{aligned}
$$




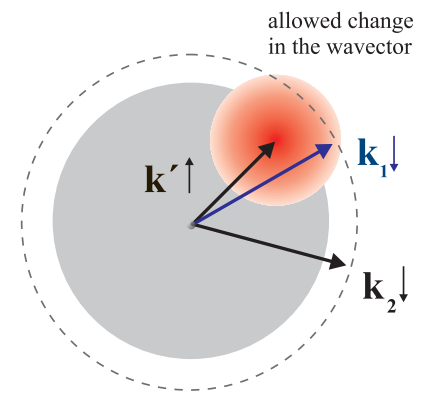

FIG. 2: (Color online) Possible spin-flip transitions. The initial state is $\overline{\mathbf{k}}^{\prime} \uparrow$, the final states are $\overline{\mathbf{k}}_{1,2} \downarrow$. Dashed circle corresponds to the transitions allowed by the energy conservation. The region in the momentum space with the size of the order of inverse correlation length $1 / R$, where the transitions can occur effectively, is also marked in the figure. If the final state is inside this area, the transition has a relatively high probability.

where $\Delta=g \mu_{B} B, g$ is the electron Lande factor, and $\mathbf{n}$ is the direction of $\mathbf{B}$. We include the magnetic field via the Zeeman term, while neglect its orbital effects. The electron energy spectrum is then spin-split, $\varepsilon_{\mathbf{k} \uparrow, \downarrow}=\varepsilon_{\mathbf{k}} \pm \Delta / 2$, where arrows correspond to the direction parallel and opposite to the magnetic field. At realistic conditions, the splitting is much smaller than the chemical potential $\mu$ of the degenerate electron gas. Here the periodic field leads to a disorder in $V_{\text {ext }}$ due to the factor $\lambda(\mathbf{r})$. As a result, $V_{\text {ext }}$ causes transitions with the change in the electron momentum and spin in a single process, as shown in Fig.2. This is in contrast to conductivity, where the coupling of the external field to the disorder appears only through the disorder effect on the electron states, and the transitions are momentum-conserving. We calculate below the corresponding spin pumping rate.

For given geometry of the external fields, denoted here as $[g]$ the time evolution of the spin projected electron density $n_{\sigma}^{[g]}$ is due to the spin-gain $I_{\sigma^{\prime} \rightarrow \sigma}^{[g]}(\omega, \Delta)$ and spin$\operatorname{loss} I_{\sigma \rightarrow \sigma^{\prime}}^{[g]}(\omega, \Delta)$ processes:

$$
\frac{d n_{\sigma}^{[g]}}{d t}=I_{\sigma^{\prime} \rightarrow \sigma}^{[g]}(\omega, \Delta)-I_{\sigma \rightarrow \sigma^{\prime}}^{[g]}(\omega, \Delta) .
$$

The concentration gain

$$
\begin{aligned}
& I_{\sigma^{\prime} \rightarrow \sigma}^{[g]}(\omega, \Delta)=2 \pi \sum_{\overline{\mathbf{k k}^{\prime}}}\left|\left\langle\sigma\left|W_{\overline{\mathbf{k k}^{\prime}}}^{[g]}\right| \sigma^{\prime}\right\rangle\right|^{2} \\
& \times\left[f\left(\varepsilon_{\overline{\mathbf{k}^{\prime}}}\right)-f\left(\varepsilon_{\overline{\mathbf{k}}}\right)\right] \delta\left(\varepsilon_{\overline{\mathbf{k}}}-\varepsilon_{\overline{\mathbf{k}^{\prime}}}-\omega\right),
\end{aligned}
$$

is due to all possible transitions from occupied $\sigma^{\prime}$ to unoccupied $\sigma$ states; a similar expression holds for the loss $I_{\sigma \rightarrow \sigma^{\prime}}(\omega, \Delta)$. The perturbation associated with the dipole moment acquired by electron spin in the presence of SO coupling ${ }^{2,3}$ has the form

$$
W_{\frac{[g]}{\mathbf{k} \mathbf{k}^{\prime}}}^{\left[\frac{e}{c}\right.} \lambda_{\mathbf{k}-\mathbf{k}^{\prime}}\left(\sigma_{y} A_{0 x}-\sigma_{x} A_{0 y}\right) .
$$

Due to charge conservation, $d\left(n_{\sigma}^{[g]}+n_{\sigma^{\prime}}^{[g]}\right) / d t=0$. Thus, pumping rate for the spin density component along the

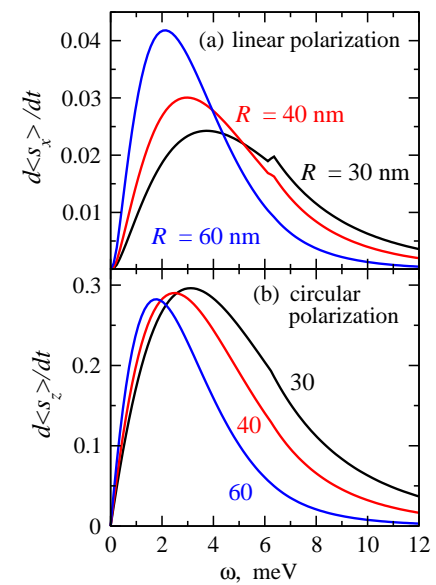

FIG. 3: (Color online) Spin pumping as a function of $\omega$ for different values of correlation radius $R$ marked near the lines. (a) In-plane field and linearly polarized radiation. Weak peaks seen at small $R$ at $\omega=\mu$ are manifestations of the spin-split density of states. We use units $(d\langle s\rangle / d t)_{0}=1 / \pi \times\left(e^{2} / c^{2}\right) A_{0 x}^{2} / \tau_{s 0}$. (b) $z$-axis field with $B \rightarrow 0$ and circularly polarized radiation. We use units $(d\langle s\rangle / d t)_{0}=2 / \pi \times\left(e^{2} / c^{2}\right) A_{0}^{2} / \tau_{s 0}$.

magnetic field $\left\langle s_{\mathbf{B}}^{[g]}\right\rangle$, is

$$
\frac{d\left\langle s_{\mathbf{B}}^{[g]}\right\rangle}{d t}=\frac{d}{d t} \frac{n_{\uparrow}^{[g]}-n_{\downarrow}^{[g]}}{2}=\frac{d n_{\uparrow}^{[g]}}{d t} .
$$

Substituting (18) into (17) and averaging over spin disorder, we obtain the component of the generation rate

$$
\begin{aligned}
& I_{\sigma^{\prime} \rightarrow \sigma}(\omega, \Delta)=2 \pi \frac{e^{2}}{c^{2}} K_{\sigma^{\prime} \rightarrow \sigma}^{[g]} \sum_{\mathbf{k k}^{\prime}} C_{\lambda \lambda}(\mathbf{q}) \\
& \times\left[f\left(\varepsilon_{\overline{\mathbf{k}}^{\prime}}\right)-f\left(\varepsilon_{\overline{\mathbf{k}}^{\prime}}+\omega\right)\right] \delta\left(\varepsilon_{\overline{\mathbf{k}}}-\varepsilon_{\overline{\mathbf{k}}^{\prime}}-\omega\right),
\end{aligned}
$$

where the coefficient $K_{\sigma^{\prime} \rightarrow \sigma}^{[g]}$ is determined by the field configuration. Here we consider two geometries: (i) Inplane magnetic field $\mathbf{B}=(B, 0,0),\left\langle s_{\mathbf{B}}^{[g]}\right\rangle=\left\langle s_{x}\right\rangle$, linearly polarized radiation $\mathbf{A}_{0}=\left(A_{0 x}, A_{0 y}\right)$ and (ii) $z$ axis magnetic field $\mathbf{B}=(0,0, B),\left\langle s_{\mathbf{B}}^{[g]}\right\rangle=\left\langle s_{z}\right\rangle$, circularly polarized radiation $\mathbf{A}_{0}=A_{0}(\mathbf{x}, i \mathbf{y})$. In the case (i) $K_{\sigma^{\prime} \rightarrow \sigma}^{[g]}=A_{0 x}^{2}$. The transition rates satisfy symmetry relation $I_{\sigma^{\prime} \rightarrow \sigma}(\omega, \Delta)=I_{\sigma \rightarrow \sigma^{\prime}}(\omega,-\Delta)$, and $d n_{\sigma}^{[g]} / d t$ in Eq. (16) vanishes if $B=0$. In the case (ii) $K_{\sigma^{\prime} \rightarrow \sigma}^{[g]}=0$ for the transitions up-down and $K_{\sigma^{\prime} \rightarrow \sigma}^{[g]}=2 A_{0}^{2}$ for the opposite process. Therefore, for the circularly polarized radiation the spin pumping occurs even at $B \rightarrow 0$.

The exact formula for the pumping rate, valid in the general case $\mu>\Delta / 2$, can be obtained after integrating Eq.(20) over the directions of $\mathbf{q}=\mathbf{k}-\mathbf{k}^{\prime}$. As a result one obtains (with $y=k R$ )

$$
I_{\sigma^{\prime} \rightarrow \sigma}(\omega, \Delta)=\frac{2}{\pi} \frac{e^{2}}{c^{2}} K_{\sigma^{\prime} \rightarrow \sigma}^{[g]} \frac{1}{\tau_{s 0}} \int_{y_{\min , \sigma^{\prime}}}^{y_{\max , \sigma^{\prime}}} y d y
$$




$$
\times \int_{\left|y-y_{\sigma^{\prime}}\right|}^{y+y_{\sigma^{\prime}}} \frac{e^{-x} x d x}{\left[4 y^{2} x^{2}-\left(x^{2}+2 m\left(\sigma^{\prime} \Delta-\omega\right) R^{2}\right)^{2}\right]^{1 / 2}},
$$

where $y_{\min , \sigma^{\prime}}=R\left[\max \left\{0,2 m\left(\mu-\sigma^{\prime} \Delta / 2-\omega\right)\right\}\right]^{1 / 2}$, $y_{\max , \sigma^{\prime}}=R\left[2 m\left(\mu-\sigma^{\prime} \Delta / 2\right)\right]^{1 / 2}, y_{\sigma^{\prime}}=\left[y^{2}+2 m(\omega-\right.$ $\left.\left.\sigma^{\prime} \Delta\right) R^{2}\right]^{1 / 2}$, and $1 / \tau_{s 0}$ is the prefactor in Eq. (12).

For numerical calculations we use the following parameters: electron effective mass for a Si/Ge (001) QW $m=0.19 m_{0}$ (where $m_{0}$ is free electron mass), electron concentration per valley $n=5 \times 10^{11} \mathrm{~cm}$, Fermi momentum $k_{F}=1.8 \times 10^{6} \mathrm{~cm}^{-1}$, and the Fermi energy $\mu=6.3 \mathrm{meV}$. To calculate $d\left\langle s_{x}\right\rangle / d t$ we take magnetic field $B=1 \mathrm{~T}$ leading to the spin splitting $\Delta=0.12 \mathrm{meV}$ for $g=2$. The injection rates $d\left\langle s_{\mathbf{B}}^{[g]}\right\rangle / d t$ are presented in Fig. 3. For both radiation polarizations, the peaks have the maximum position and the width on the order of $\omega \sim \mu / k_{F} R$. With the increase in $R$ at given $k_{F}$, the available momentum and energy ranges decrease, the peaks sharpen and shift to lower frequencies. For linearly polarized radiation (Fig. 3(a)), the pumping rate is linear in $\Delta$.
Conclusions We have studied spin relaxation and infrared radiation-induced spin transitions in a $2 \mathrm{D}$ electron gas with the Rashba field disorder. Quantum effects related to noncommutativity of the momentum and random Rashba potential lead to the decrease in the spin relaxation rate when the spatial scale of the randomness is smaller than the electron wavelength. In contrast to conductivity, external periodic electromagnetic field generates a perturbation directly including the SO disorder, and, therefore, causing spin-flip accompanied by a momentum change. As a result, electron spin density can be pumped by coupling of spins to the external periodic field in the frequency range up to the Fermi energy. These effects extend the abilities of manipulating spins in semiconductor structures.

Acknowledgements. This work is partly supported by the FCT Grant PTDC/FIS/70843/2006 in Portugal and by Polish Ministry of Science and Higher Education as a research project in years 2007 - 2010. E. Sherman acknowledges support of the University of Basque Country UPV-EHU grant GIU07/40 and valuable discussion with M.M. Glazov.
1 I. Zutic, J. Fabian, and S. Das Sarma, Rev. Mod. Phys. 76, 323 (2004)

2 E.I. Rashba and V.I. Sheka, Fizika Tverd. Tela (Leningrad) 3, 2369 (1961) [Sov. Phys. Solid State 3, 1718 (1962)]; E.I. Rashba and V.I. Sheka, in: G. Landwehr and E.I. Rashba, Editors, Landau Level Spectroscopy, North-Holland, Amsterdam (1991), p. 131 and references therein.

3 E. I. Rashba and Al. L. Efros, Phys. Rev. Lett. 91, 126405 (2003); Appl. Phys. Lett. 835295 (2003).

4 M. Duckheim and D. Loss, Phys. Rev. Lett. 101, 226602 (2008).

5 D. V. Khomitsky, Phys. Rev. B 77, 113313 (2008); Phys. Rev. B 79, 205401 (2009).

${ }^{6}$ K. C. Nowack, F. H. L. Koppens, Yu. V. Nazarov, L. M. K. Vandersypen, Science 318, 1430 (2007).

7 M. Pioro-Ladriere, T. Obata, Y. Tokura, Y.-S. Shin, T. Kubo, K. Yoshida, T. Taniyama, S. Tarucha, Nature Physics 4, 776 (2008).

8 Y. J. Bao and S. Q. Shen, Phys. Rev. B 76, 045313 (2007).

9 E. Ya. Sherman, Phys. Rev. B 67, 161303(R) (2003).

10 M. M. Glazov and E. Ya. Sherman, Phys. Rev. B 71, 241312(R) (2005). If the Rashba field is produced by the random donors, $\left\langle\lambda^{2}\right\rangle$ behaves as $1 / R^{2}$, and, therefore, the spin relaxation time decreases with the increase in $R$.

11 L. E. Golub and E. L. Ivchenko, Phys. Rev. B bf 69, 115333 (2004)

12 C. P. Moca, D. C. Marinescu, and S. Filip, Phys. Rev. B 77, 193302 (2008).

13 Y. Tserkovnyak and S. Akhanjee, Phys. Rev. B 79, 085114 (2009).
14 Z. Wilamowski, W. Jantsch, H. Malissa, and U. Rössler, Phys. Rev. B 66, 195315 (2002).

15 M.-H. Liu, K.-W. Chen, S.-H. Chen, and C.-R. Chang Phys. Rev. B 74, 235322 (2006).

16 J. D. Koralek, C. P. Weber, J. Orenstein, B. A. Bernevig, Shou-Cheng Zhang, S. Mack, and D. D. Awschalom, Nature 458, 610 (2009).

17 G. M. Müller, M.l Römer, D. Schuh, W. Wegscheider, J. Hübner, and M. Oestreich, Phys. Rev. Lett. 101, 206601 (2008).

18 V. V. Bel'kov, P. Olbrich, S. A. Tarasenko, D. Schuh, W. Wegscheider, T. Korn, C. Schüller, D. Weiss, W. Prettl, and S. D. Ganichev, Phys. Rev. Lett. 100, 176806 (2008).

19 D. Huertas-Hernando, F. Guinea, and A. Brataas, Eur. Phys. J. Special Topics 148, 177 (2007).

20 C. Ertler, S. Konschuh, M. Gmitra, and J. Fabian, Phys. Rev. B 80, 041405 (2009).

21 S.A. Tarasenko, Pis'ma v ZhETF 84, 233 (2006) [JETP Letters 84, 199 (2006)].

22 D. Culcer and R. Winkler, Phys. Rev. B, 76, 195204 (2007); D. Culcer, preprint cond-mat arXiv:0904.1999 (2009); R. Winkler, D. Culcer, S. J. Papadakis, B. Habib, and M Shayegan, Semicond. Sci. Technol. 23, 114017 (2008).

23 M. M. Glazov, Sol. State Commun. 142, 531 (2007); Phys. Rev. B 70, 195314 (2004).

24 T. Ando, A. B. Fowler, and F. Stern, Rev. Mod. Phys. 54, 437 (1982). 